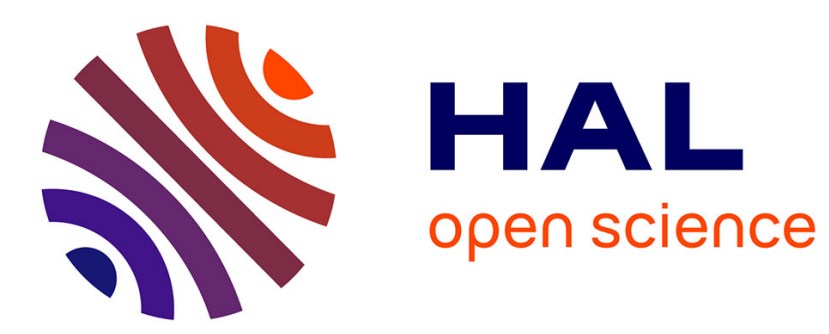

\title{
Ionic collisions in metallic lattices under transient current pulses
}

\author{
C. D. Papageorgiou, T. E. Raptis
}

\section{To cite this version:}

C. D. Papageorgiou, T. E. Raptis. Ionic collisions in metallic lattices under transient current pulses. European Physical Journal: Applied Physics, 2011, 54 (1), 10.1051/epjap/2011100325 . hal00687328

\section{HAL Id: hal-00687328 \\ https://hal.science/hal-00687328}

Submitted on 13 Apr 2012

HAL is a multi-disciplinary open access archive for the deposit and dissemination of scientific research documents, whether they are published or not. The documents may come from teaching and research institutions in France or abroad, or from public or private research centers.
L'archive ouverte pluridisciplinaire HAL, est destinée au dépôt et à la diffusion de documents scientifiques de niveau recherche, publiés ou non, émanant des établissements d'enseignement et de recherche français ou étrangers, des laboratoires publics ou privés. 
Dear Prof. Luis Lemos Alves

Having followed carefully the recommendations provided by the referee, we have updated the article accordingly.

Taking the opportunity, we would like to express our thanks to the referee for his careful reading of the manuscript and his help for optimizing the presentation of our results. Attached is the new, updated version of our article for your consideration. We are looking forward to your comments and further recommendations. 


\title{
Ionic Collisions in Metallic Lattices under Transient Current Pulses
}

\author{
C. D. Papageorgiou ${ }^{1}$, T. E. Raptis ${ }^{2}$ \\ ${ }^{1}$ Dept. of Electrical \& Electronic Engineering, \\ National Technical University of Athens, Greece \\ E-mail: chrpapa@ central.ntua.gr \\ ${ }^{2}$ Division of Applied Technologies, \\ National Centre for Science and Research "Demokritos", \\ Patriarchou Grigoriou \& Neapoleos, Athens, Greece \\ E-mail: rtheo@dat.demokritos.gr
}

\begin{abstract}
A novel method for ionic collisions is presented based on a theoretical analysis of the long-standing wire fragmentation problem. Based on a Maxwellian approach of the transient electrodynamic forces we deduce a theoretical formula of the total effective tensile force. A number of conducted experiments with low energy capacitor bank discharges are also reported with positive results. The new results show the possibility of building solid state ion colliders below the fragmentation threshold using high power electrical pulses with very low energy.
\end{abstract}

Keywords: Ion Collisions, Metallic lattice, Current pulses

PACS Code : 077.50.Ek, 29.20.db, 63.20.dd

\section{Introduction}

In a series of previous publications $[1,2]$ we analyzed the problem of wire fragmentation, which is associated with a long-standing controversy in Maxwellian electrodynamics. The first experiments in wire explosions under very high currents and voltages were made by Nasilowsky during the 60s. At the 80s, Pappas [3], Graneau [4, 5] and Rambaut and Vigier [6] repeated similar experiments and argued on the existence of longtitudinal forces beyond the ordinary Maxwellian electrodynamics. Other attempts for a correct explanation $[7,8]$ fail to provide a clear picture of the phenomena in terms of the Maxwell stress tensor.

A corrected picture in terms of the classical Maxwell theory is presented which is amenable to experimental verification in section 2, based on the instantaneous forces between radiating dipoles created by the action of a transient high power current pulse. In section 3, experimental results are reported with a particular pulse generator of very low internal resistance and low capacitor energy. Results with this method show impressive alterations and fatigue exerted on the metallic structure of thin wires even below the fragmentation threshold. In section 4, it is proposed to use similar effects in order to create a solid state ion collider. Arguments are presented for the treatment of special metals and/or unstable isotopes by this method for low energy nuclear transmutations and possibly for neutralization of radio wastes.

\section{Macroscopic treatment of forces on thin wires under transient standing waves}


Let us consider a thin linear conductor (hereafter termed "wire") of length $L$ along the $\mathrm{z}$-axis and centered in the origin. Let us assume also that on this thin wire a short duration electric pulse current is excited through the discharge of a capacitor. This current pulse has a transient behavior in the initial very short duration time of his application, considering the thin wire as an antenna or an electric line. The result of the current pulse on the thin wire will be a set of transient space harmonic standing waves related to its length $L$. Due to the assumption that the wire is very thin we can also assume that the first (or second) fundamental transient standing electromagnetic wave has a wave length equal to $L$ and a frequency $L / c$ where $c=3 \cdot 10^{8} \mathrm{~m} / \mathrm{sec}$.

Then the current of this transient standing electromagnetic wave current along the $\mathrm{z}$ axis will be given as

$$
I(z, t)=\operatorname{Re} \sqrt{2} I \exp (\mathbf{i} \omega t) \sin (k z)
$$

where $k=2 \pi / L, \omega=c \cdot k, L / 2 \geq z \geq-L / 2$, i.e., $\pi \geq k z \geq-\pi$. If we divide the thin wire considering it as an antenna into small infinitesimal dipoles of length $d z_{1}$ then all dipoles from the first half wavelength (upper part of the antenna) are oppositely oriented to the rest of the dipoles for the other half wavelength.

The main assumptions behind our model is that the transient release of the current pulse leads to violent reorientation of the internal structure of the lattice charges which then subside before entering a steady state flow. The associated effects can be approximated by the use of the infinitesimal ideal (Hertzian) dipoles in the near field regime to express the dynamics of the overall transient polarization field developed inside the conductor's lattice. The main calculation of the forces between the dipoles due to radiation effects is presented in Appendix A. The main result for the interdipole force is summarized in the formula (A17) or

$$
d F_{12}=-\frac{180}{c} \sin \left(k z_{1}\right) \sin \left(k z_{2}\right) \Phi\left(k z_{12}\right) d\left(k z_{1}\right) d\left(k z_{2}\right)
$$

where as found in App. A $\Phi(\chi)=-\left[\left(\frac{1}{\chi^{4}}-\frac{1}{3 \chi^{2}}\right) \cos \chi+\frac{1}{\chi^{3}} \sin \chi\right]$

The actual distance between two opposite dipoles $d z_{1}$ and $d z_{2}$ is approximately equal for small diameters in comparison to wire length as:

$$
\left|x_{1}-x_{2}\right| \approx \sqrt{\left(x_{1}-x_{2}\right)^{2}+(k D)^{2}}, k D=2 \pi D / L
$$

We should notice that when the length $x_{1}$ and $x_{2}$ become comparable to the diameter $\mathrm{D}$ the distance between adjacent dipoles $\left|x_{1}-x_{2}\right|$ should be calculated with a smaller effective diameter. This could be incorporated with an appropriate empirical coefficient such that $D_{\text {eff }}=c_{1} D, c_{1}<1$. Then the overall repelling force will be given approximately by: 


$$
F_{12}=-\frac{180}{c} I^{2} \int_{0}^{\pi} d x \sin x_{1} \int_{\pi}^{2 \pi} d x_{2} \sin x_{2} \Phi\left(x_{1}-x_{2}\right)
$$

or

$$
F_{12}=-\frac{180 I^{2}}{c} \varphi(k D)
$$

In the following graph (2) the steady repulsive force acting on the lower L/2 part of the thin wire by all the upper dipoles of its upper length $\mathrm{L} / 2$ is shown as function of the ratio of the wave length to equivalent wire diameter. The integral $\varphi(k D)$ was numerically evaluated in MATLAB standing for a "Tensile Force Factor" and the force was calculated with respect to the parameter $L / D=2 \pi / k D$ as shown in Fig. 2. Thus the "bipolar" repelling force between the upper and lower parts of a wire of a given length can be calculated for given values of the diameter.

Using the same approach the repelling force on the set of dipoles around $d x$ in one half of the wire in a distance $x_{1}$ off the centre of the wire (calculated in wire diameters) arising by all the dipoles of the other half of the wire can be found as

$$
F\left(x_{1}\right)=-\frac{180}{c} I^{2} \sin x_{1} \int_{\pi}^{2 \pi} d x_{2} \sin x_{2} \Phi\left(x_{1}-x_{2}\right)
$$

This function was numerically evaluated for various values of $x_{1}$ (in wire diameters) and its values (as a percentage of the maximum value) are shown in Fig. 3 for a wire length equal to 200 wire diameters.

From the data of Fig. 3 it is evident that the maximum repelling force is exercised on the dipoles near the centre of the wire. That explains the expectation that the first crack on the wire due to dipolar repelling force will appear near the centre of the wire. It also agrees with experimental evidence from the heavy water drill apparatus where half of the heavy water is expelled during its operation [13]. From what appears to be the case, the quantity of the expelled water leaving the drill tube comes from the second half of the tube and gets separated near the middle of the tube while the other half which is pushed backwards remains in the interior of the tube.

\section{Experimental investigation of bipolar forces}

In order to prove the existence of strong ionic collisions inside thin wires a special pulse generator was designed. The basic circuit is shown in Fig. 4. The aim behind this design was to have the overall internal resistance of this circuit to lie in a range of some $\mathrm{m} \Omega$ 's. For this, a special set of capacitors and a thyristor of very low internal resistance were chosen.

The current pulses were created by the discharge of the capacitor bank composed of 4 cpacitors in parallel, each of $3 \mu \mathrm{F}$ with $12 \mu \mathrm{F}$ total capacitance (4.3), with the aid of a special digital controller circuit (4.6) that allowed both a single pulse or a sequence of consecutive pulses (see Fig. 4). The actual device is shown in Fig. 5. The duration between subsequent pulses in the sequential mode was of the order of 3 secs. These 
were then applied to a variety of wires of variable diameters and a constant length of approximately $27 \mathrm{~cm}$.

The exercised voltage of the capacitors was nearly $1000 \div 1200$ Volts and the discharge resistances including the circuit and the additional wire resistance were from $100 \mathrm{~m} \Omega$ to $300 \mathrm{~m} \Omega$. The time constant of the occurring discharges were thus in the range of several $\mu$ secs.

The values of the instantaneous currents exerted on the wires from these current pulses were in the range of $4000 \mathrm{~A}-12000 \mathrm{~A}$. It was not possible to measure the magnitude of the fundamental harmonic but its existence could be detected by the appearance of longitudinal vibration of the thin wires that could be detected visually and easily could be captured by a high resolution camera.

Direct inspection showed that during the first pulse, a cloud of metallic dust gets violently expelled from the wire surface. This is speculated to be the result of the bipolar forces on defects and dislocations in the metallic crystal lattice. After several pulses depending on the wire diameter some of the wires get cut not necessarily near the center of the total length. This apparent discrepancy with the theoretical prediction of section 2 is probably due to the simultaneous coexistence of several different wavelengths due to the continuous spectrum of the discharge curve.

A number of wires with a steel core and a conductive metallic layer deposited with an electrolytic bath were also tried to show that it is possible to increase the tensile strength to avoid fragmentation. Indeed, the type of fatigue of the conductive metallic layer remained the same although the wire did not get cut despite a large number of pulses applied sequentially for a long time interval of several minutes up to half an hour.

In particular, the specific type of fatigue appearing in a set of experiments with copper wires of $27 \mathrm{~cm}$ length and an average resistance of $300 \mathrm{~m} \Omega$ was shown to be characterized by three major effects.

a) A number of almost periodic burns on the external surface of the wire that is probably associated with the wavelengths of higher harmonics.

b) An overall zig-zag shape obtained by a loose wire and a subsequent length shortening that is strongly suggestive of the opposite local dipole polarities ,due to the action of several harmonics, at certain sections of the wire.

c) At some of the experiments sparks appeared to emanate from points of the surface totally unrelated with the final break point. Some of these were captured with the video camera and may be related with local charge concentrations on previously created cracks of the metal surface.

Observation of the condition of the wires surface took place at the Laboratory of Metallurgical Microscopy of the National Technical University of Athens. Results were photographed and are shown in the pictures of Fig. 6. Examination of these samples revealed both vertical as well as helical cracks on the metal surface.

Another question concerning the appearance of the characteristic "neck"-like configuration of a metal near the break point due to increased metal plasticity as usually predicted was not verified. The final breaking mechanism may require separate study but our purpose in these experiments was rather to prevent the wires from reaching their tensile strength threshold in order to create a sustainable mechanism for exerting bipolar forces on a metallic lattice without breaking.

\section{The possibility of transmutations inside metallic lattices under high current pulses}


During the last thirty years various experimenters [10 - 14] have found evidence of $\mathrm{X}$-rays and neutron production during fragmentation tests. In the most recent case, Shyam and Rout [14] used Palladium Deuteride in a discharge experiment and reported an outflux of $0.5 \times 10^{4}$ neutrons per discharge.

The difference in the experiments reported here lies in the fact that we used a very low internal resistance circuit with a minimum energy stored in the capacitor bank not exceeding 20J. This allows the maximum current flow with a minimum of dissipated energy in order to reach a practical method for energy production without fragmenting the used conductors that play the role of the fuel.

The question remaining to be answered concerns the use of other metallic compounds with different types of nuclear chemistry that could exhibit either fission or fusion reactions. The unique interpretation offered here shows that the existence of stationary waves across the conductor's body moves the ionic centres in an inhomogeneous manner. It is then expected that certain "hot spots" will appear on the metallic surface depending of the type of metal used due to the ionic collisions concentrated around the nodal points of the system of stationary waves where opposite polarities of the local radiating dipoles meet.

According to the analysis presented in section 2 the forces exerted on the lattice depend on the total current flowing through the conductor and not in the energy stored in the capacitor bank. This distinguishes our approach to the problem form other similar approaches where huge amounts of energy of the order of several $\mathrm{kJ}$ are thrown in the wires like in Z-pinch or X-pinch experiments.

At first approximation and for low energies we may approximate the situation on the conductor with a standard lattice model where the fed energy gets dispersed among a system of normal modes or phonons that propagate with different group velocities. In order for a transmutation to occur it is necessary that an amount of ions will gain enough energy to overcome the Coulomb barrier. The probability for this to happen depends strongly on the phonon density of states $g(\mathrm{E})$. Assuming a boson statistics this will be given approximately from the quantity $f(\mathrm{E}) g(\mathrm{E})$ where the distribution $f(E)=g_{i}\left[\mathrm{xp}\left(\varepsilon_{i}-\mu\right) / k T-1\right]$ depends on the chemical potential $\mu$ of the metal used. The reaction rate near the excitation energies $\varepsilon_{l}$ of unstable nuclei depends on the particular shape of the $g(\mathrm{E})$ curve.

Estimation of $g(\mathrm{E})$ is not trivial for the case of a very strong transient electric pulse. Although some studies with $f s e c$ laser pulses exist in the literature, the situation of a whole conductor is mostly difficult to analyze theoretically when the applied external field varies in both space and time as in the case of a system of transient stationary waves. Anharmonicities will also appear due to the presence of a vector potential in the Hamiltonian of the ionic lattice that gets also affected by secondary emissions as the ions move violently in the opposite direction of the electron gas to conserve momentum. It is even possible that above a certain threshold of the applied voltage there will be deviations from the assumed linearity (purely Ohmic resistance) of the current - voltage (I-V) curve due to nonlinearities present in a macroscopic sample.

Despite the theoretical difficulties all experimental evidence supports the possibility of low energy transmutations in the presence of high power electrical pulses in linear conductors. Our goal in these and subsequent experiments will be to try different metallic compounds of special unstable elements to find out such combinations that will be sufficient for nuclear reactions with the minimum possible energy input.

\section{Conclusions}


We have presented both theoretical and experimental evidence of ionic collisions inside metallic lattices of linear conductors when excited by high power and low energy electrical pulses. Based on several previous experimental attempts we build an explanatory macroscopic physical model based on classical Maxwell theory for the transient electromagnetic forces exerted on the ionic centers that allows experimental verification. In contrast with previous efforts, we show by direct experimental evidence that low energy, high power pulses can be used to achieve strong enough forces on the conductors without fragmentation. We also propose to use them for controllable nuclear transmutations in the case of special or unstable metallic compounds for a variety of possible uses that range from energy production to radio waste disposal.

\section{Aknowledgments}

We would like to express our thanks to Prof Vasiliou Panagiota of NTUA for her support in preparing the wire photos in the Laboratory of Metallurgical Microscopy of the National Technical University of Athens and to the electronic engineer George Bindarchas CEO of "ELCON Systems \& Components" for the construction of the electric pulse generator and his support during the experiments.

\section{References}

[1] C. D. Papageorgiou. T. E. Raptis, Eur. Phys. J. Appl. Phys. 48, 31002 (2009)

[2] C. D. Papageorgiou. T. E. Raptis, 7th Conf. Balkan Physics Union, AIP Conf. Proceedings, (2009) 955 - 960

[3] P. T. Pappas, P. G. Moyssides, Phys. Let. A, 111(4), (1985) 193 - 198

[4] P. Graneau, Phys. Let. A, 107(5), (1985) 235 - 237

[5] P. Graneau, N. Graneau, Phys. Let. A, 174(5-6), (1993) 421 - 427

[6] M. Rambaut, J. P. Vigier, Phys. Let. A, 148(5), (1990) 229 - 238

[7] S. Molokov, D.P. Wall, J.E. Allen, J. Phys. D: Appl. Phys. 36, (2003) 2757

[8] F. Winterberg, Annales de la Fondation Louis de Broglie 31, (2006) 363

[9] J. D. Jackson, “Classical Electrodynamics”, J. Wiley (1975)

[10] W. Lochte-Holtgreven, Atomkernenergie 28 (1976) 150

[11] S. K. Handel, O. Johnson, Atomkernenerg. Kerntech. 36 (1980) 170

[12] P. Graneau, N. Graneau, Phys. Lett. A, 165(1), (1992) 1-13

[13] J. D. Sethian et al., Phys. Rev. Lett. 59 (1987) 892 
[14] A. Shyam, R. K. Rout, IEEE Trans. Plasma Sci. 27(4), (1999) 1210 - 1213

[15] W. Jones, N. H. March, “Theoretical Solid State Physics”, J. Wiley (1973)

[16] P. L. Taylor, “A Quantum Approach to the Solid State”, Prentice-Hall (1970)

[17] R. Bader, “Atoms in Molecules: A Quantum Theory”, Oxford University Press (1994)

\section{Appendix A}

The currents of the dipoles $d z_{1}, d z_{2}$ will be given by the expression

$$
I\left(z_{1}, t\right)=\operatorname{Re}\left[\sqrt{2} I \exp (\mathbf{i} \omega t) \sin \left(k z_{1}\right)\right], I\left(z_{2}, t\right)=\operatorname{Re}\left[\sqrt{2} I \exp (\mathbf{i} \omega t) \sin \left(k z_{2}\right)\right]
$$

thus their complex equivalents (considering them as having zero phase) will be given by the functions $I\left(z_{1}\right)=I \cdot \sin \left(k z_{1}\right), I\left(z_{2}\right)=I \cdot \sin \left(k z_{2}\right)$. These electric dipoles of length $d z_{1}$ and $d z_{2}$ can be represented also by their equivalent electric charges given by the relations

$$
q\left(z_{1}, t\right)=\operatorname{Re}\left[\sqrt{2} I \exp (\mathbf{i} \omega t) \sin \left(k z_{1}\right) / \mathbf{i} \omega\right], q\left(z_{2}, t\right)=\operatorname{Re}\left[\sqrt{2} I \exp (\mathbf{i} \omega t) \sin \left(k z_{2}\right) / \mathbf{i} \omega\right]
$$

Thus their complex equivalents (as having phases $-\pi / 2$ ) will be given by the functions

$$
q\left(z_{1}\right)=I \cdot \sin \left(k z_{1}\right) / \mathbf{i} \omega, q\left(z_{2}\right)=I \cdot \sin \left(k z_{2}\right) / \mathbf{i} \omega
$$

We may then assume that every such dipole $d z_{1}$ creates an electric field $d E_{12}(z)$ affecting every dipole $d z_{2}$ at the lower part of the wire and at distance $z_{12}$. We show two oppositely oriented dipoles on the wire schematically in Fig. 1.

According to standard formulas for radiating dipoles [8] we can write the total field of each elementary dipole in space as:

$$
\begin{aligned}
& E_{r}=Z_{0} \frac{2 I k^{2} d z}{4 \pi} \cos \theta(\mathbf{i} k r)^{-2}+(\mathbf{i} k r)^{-3} e^{-\mathbf{i} k r} \\
& E_{\theta}=Z_{0} \frac{I k^{2} d z}{4 \pi} \sin \theta(\mathbf{i} k r)^{-1}+(\mathbf{i} k r)^{-2}+(\mathbf{i} k r)^{-3} e^{-\mathbf{i} k r}
\end{aligned}
$$

where $Z_{0}=\sqrt{\mu_{0} / \varepsilon_{0}} \cong 120 \pi$ the impedance of free space. Along the z-axis where $\theta$ $=0$ and $k r=k z_{12}$, we find the total contribution of $d z_{1}$ to $d z_{2}$ as

$$
d E_{12}=2 Z_{0} \frac{I\left(z_{1}\right) k^{2} d z_{1}}{4 \pi}\left[\left(\mathbf{i} k z_{12}\right)^{-2}+\left(\mathbf{i} k z_{12}\right)^{-3}\right] e^{-\mathbf{i} k z_{12}}
$$


This complex function represents the real function of time $d E_{12}(t)$ and its partial derivative given as

$$
\begin{aligned}
& d E_{12}(t)=\operatorname{Re}\left[\sqrt{2} \cdot d E_{12} \cdot \exp (\mathbf{i} \omega t)\right] \\
& \frac{\partial\left(d E_{12}(t)\right)}{\partial\left(k z_{12}\right)}=\operatorname{Re}\left[\sqrt{2} \cdot \frac{\partial\left(d E_{12}\right)}{\partial\left(k z_{12}\right)} \cdot \exp (\mathbf{i} \omega t)\right]
\end{aligned}
$$

The instantaneous net Coulomb force due to $d E_{12}(t)$ on the dipole $d z_{2}$ (represented by the charges $\pm \mathrm{q}\left(\mathrm{z}_{2}\right)$ ) is given by

$$
\begin{aligned}
& d F_{12}(t)=\frac{\partial\left(d E_{12}(t)\right)}{\partial z_{12}} \cdot d z_{2} \cdot q\left(z_{2}, t\right)=k \cdot d z_{2} \cdot \frac{\partial\left(d E_{12}(t)\right)}{\partial\left(k z_{12}\right)} \cdot q\left(z_{2}, t\right) \text { i.e. } \\
& d F_{12}(t)=\operatorname{Re}\left[\sqrt{2} \cdot k \cdot d z_{2} \cdot \frac{\partial\left(d E_{12}\right)}{\partial\left(k z_{12}\right)} \cdot \exp (\mathbf{i} \omega t)\right] \cdot \operatorname{Re}\left[\sqrt{2} \cdot q\left(z_{2}\right) \cdot \exp (\mathbf{i} \omega t)\right] i . e . \\
& d F_{12}(t)=\operatorname{Re}\left[\sqrt{2} \cdot k \cdot d z_{2} \cdot \frac{\partial\left(d E_{12}\right)}{\partial\left(k z_{12}\right)} \cdot \exp (\mathbf{i} \omega t)\right] \cdot \operatorname{Re}\left[\sqrt{2} \cdot\left(I\left(z_{2}\right) / \mathbf{i} \omega\right) \cdot \exp (\mathbf{i} \omega t)\right]
\end{aligned}
$$

Using the following identity for complex numbers A and B:

$$
\operatorname{Re}[\sqrt{2} \cdot A \cdot \exp (\mathbf{i} \omega t)] \cdot \operatorname{Re}[\sqrt{2} \cdot B \cdot \exp (\mathbf{i} \omega t)]=\operatorname{Re}\left[A \cdot B^{*}\right]+\operatorname{Re}[A \cdot B \cdot \exp (\mathbf{i} 2 \omega t)]
$$

it can be shown that

$$
d F_{12}(t)=\operatorname{Re}\left[k \cdot d z_{2} \cdot \frac{\partial\left(d E_{12}\right)}{\partial\left(k z_{12}\right)} \cdot\left(I^{*}\left(z_{2}\right) /-\mathbf{i} \omega\right)\right]+\operatorname{Re}\left[k \cdot d z_{2} \cdot \frac{\partial\left(d E_{12}\right)}{\partial\left(k z_{12}\right)} \cdot\left(I\left(z_{2}\right) / \mathbf{i} \omega\right) \cdot \exp (\mathbf{i} 2 \omega t)\right]
$$

Taking into consideration that $I^{*}\left(z_{2}\right)=I\left(z_{2}\right)=I \cdot \sin \left(k z_{2}\right)$ and $k / \omega=1 / c$ we can deduce that the instantaneous force between the dipoles 1 and 2 is comprising of two parts a constant repelling force and an instantaneous sinusoidal force. The constant repelling force is given by the formula

$$
\left.d F_{12}=\operatorname{Re}\left[k \cdot d z_{2} \cdot \frac{\partial\left(d E_{12}\right)}{\partial\left(k z_{12}\right)} \cdot\left(I \cdot \sin \left(k z_{2}\right)\right) /-\mathbf{i} \omega\right)\right]=-\frac{I \cdot \sin \left(k z_{2}\right)}{c} \cdot d z_{2} \cdot \operatorname{Jmag}\left[\frac{\partial\left(d E_{12}\right)}{\partial\left(k z_{12}\right)}\right]
$$

Taking into consideration that

$$
\operatorname{Jmag}\left[d E_{12}\right]=Z_{0} \cdot k^{2} \frac{I \cdot \sin \left(k z_{1}\right) d z_{1}}{2 \pi}\left[\frac{\cos \left(k z_{12}\right)}{\left(k z_{12}\right)^{3}}+\frac{\sin \left(k z_{12}\right)}{\left(k z_{12}\right)^{2}}\right]
$$

The force differentials are derived as 


$$
\begin{aligned}
& d F_{12}=-\left(\frac{1}{c}\right) 60 k^{2} I^{2} \sin \left(k z_{1}\right) \sin \left(k z_{2}\right) \frac{\partial f}{\partial\left(k z_{12}\right)} d z_{1} d z_{2} \\
& f\left(z_{12}\right)=\frac{\cos \left(k z_{12}\right)}{\left(k z_{12}\right)^{3}}+\frac{\sin \left(k z_{12}\right)}{\left(k z_{12}\right)^{2}}
\end{aligned}
$$

After some algebra these take the form

$$
\begin{gathered}
d F_{12}=\frac{180 \cdot I^{2}}{c} \sin \left(k z_{1}\right) \sin \left(k z_{2}\right) \Phi\left(k z_{12}\right) d\left(k z_{1}\right) d\left(k z_{2}\right) \\
\Phi(\chi)=\left[\left(\frac{1}{\chi^{4}}-\frac{1}{3 \chi^{2}}\right) \cos \chi+\frac{1}{\chi^{3}} \sin \chi\right]
\end{gathered}
$$

As a result of the above relation the overall repulsive force acting on the dipole $d z_{2}$ (on the lower $L / 2$ part of the transient antenna) by all the dipoles of the upper $L / 2$ part is given by the integral

$$
F\left(x_{2}\right)=\left[\frac{180}{c} \cdot I^{2} \cdot \sin x_{2} \cdot \int_{0}^{\pi} \sin x_{1} \cdot \Phi\left(x_{1}-x_{2}\right) \cdot d x_{1}\right] \cdot d x_{2}, x_{1}=k \cdot z_{1}, x_{2}=k \cdot z_{2}
$$

Thus the overall transient repulsive force between the lower and the upper parts of the antenna due to the first harmonic (of $L$ wave length) is given by the integral

$$
F_{12}=\frac{180}{c} \cdot I^{2} \cdot \int_{\pi}^{2 \pi} \sin x_{2} \cdot\left[\int_{0}^{\pi} \sin x_{1} \cdot \Phi\left(x_{1}-x_{2}\right) d x_{1}\right] \cdot d x_{2}
$$

The overall sinusoidal force of angular frequency $2 \omega$ has a maximum value given by the formula

$$
d F_{12}(\max )=\left|\sum_{1} \frac{I \cdot \sin \left(k z_{2}\right)}{c} \cdot \frac{\partial\left(d E_{12}\right)}{\partial\left(k z_{12}\right)}\right|
$$

Thus after some algebra it can be proved that the overall sinusoidal force acting on the dipole $d z_{2}$ (on the lower $L / 2$ part of the transient antenna) by all the dipoles of the upper $L / 2$ part can be calculated by the formula $F\left(x_{2}\right)=\sqrt{F_{1}^{2}+F_{2}^{2}}$ where

$$
\begin{aligned}
& F_{1}=\left[\frac{180}{c} \cdot I^{2} \cdot \sin x_{2} \cdot \int_{0}^{\pi} \sin x_{1} \cdot \Psi\left(x_{1}-x_{2}\right) \cdot d x_{1}\right] \cdot d x_{2} \\
& F_{2}=\left[\frac{180}{c} \cdot I^{2} \cdot \sin x_{2} \cdot \int_{0}^{\pi} \sin x_{1} \cdot \Phi\left(x_{1}-x_{2}\right) \cdot d x_{1}\right] \cdot d x_{2}
\end{aligned}
$$

where 


$$
\begin{aligned}
& \Psi(\chi)=\left[\left(\frac{1}{\chi^{4}}-\frac{1}{3 \chi^{2}}\right) \sin \chi-\frac{1}{\chi^{3}} \cos \chi\right] \\
& \Phi(\chi)=\left[\left(\frac{1}{\chi^{4}}-\frac{1}{3 \chi^{2}}\right) \cos \chi+\frac{1}{\chi^{3}} \sin \chi\right]
\end{aligned}
$$

However it can be proved that the integral $F_{2}$ is always bigger than the integral $F_{1}$, thus approximately the maximum sinusoidal component is almost equal to the steady component. This means that the maximum value of the instantaneous repelling force on the dipoles can become two times their steady values. Although the frequency of the instantaneous repelling forces is very high for light dipoles of small inertial mass the effect of the sinusoidal component could be substantial.

As a final remark we can notice that the steady repulsive force can be calculated using stored energy due to the interaction of the dipoles 1 and $2, d W_{12}$. The stored energy $d W_{12}$ is calculated through the mutual reactive power $d Q_{12}$ and the steady repulsive force is calculated through $d W_{12}$ by the relations

$$
d W_{12}=\frac{d Q_{12}}{2 \omega}, d F_{12}=-\frac{\partial\left(d W_{12}\right)}{\partial z_{12}}=-\frac{k}{2 \omega} \frac{\partial\left(d Q_{12}\right)}{\partial\left(k z_{12}\right)}
$$

The mutual reactive power is calculated from

$$
d Q_{12}=2 \operatorname{Im} \backslash E_{12}\left(z_{2}\right) I\left(z_{2}\right) d z_{2}^{-}
$$

where the factor of 2 is to take into account the two equal and opposite actions from $d z_{1}$ to $d z_{2}$ and vice versa. Thus:

$$
\begin{aligned}
& d Q_{12}=60 k^{2} I\left(z_{1}\right) I\left(z_{2}\right) f\left(z_{12}\right) d z_{1} d z_{2} \\
& f\left(z_{12}\right)=\frac{\cos \left(k z_{12}\right)}{\left(k z_{12}\right)^{3}}+\frac{\sin \left(k z_{12}\right)}{\left(k z_{12}\right)^{2}}
\end{aligned}
$$

This leads to a steady repelling force which is given as

$$
d F_{12}=-\left(\frac{1}{2 c}\right) 60 k^{2} I\left(z_{1}\right) I\left(z_{2}\right) \frac{\partial f}{\partial\left(k z_{12}\right)} d z_{1} d z_{2}
$$




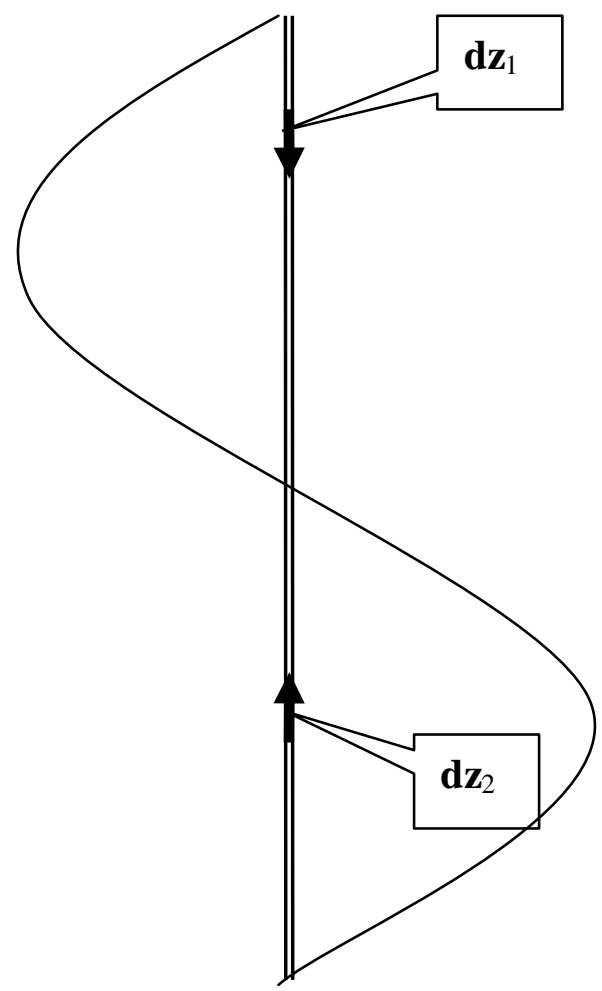

Figure 1. Schematic representation of the oppositely oriented instantaneous dipoles on the thin wire 


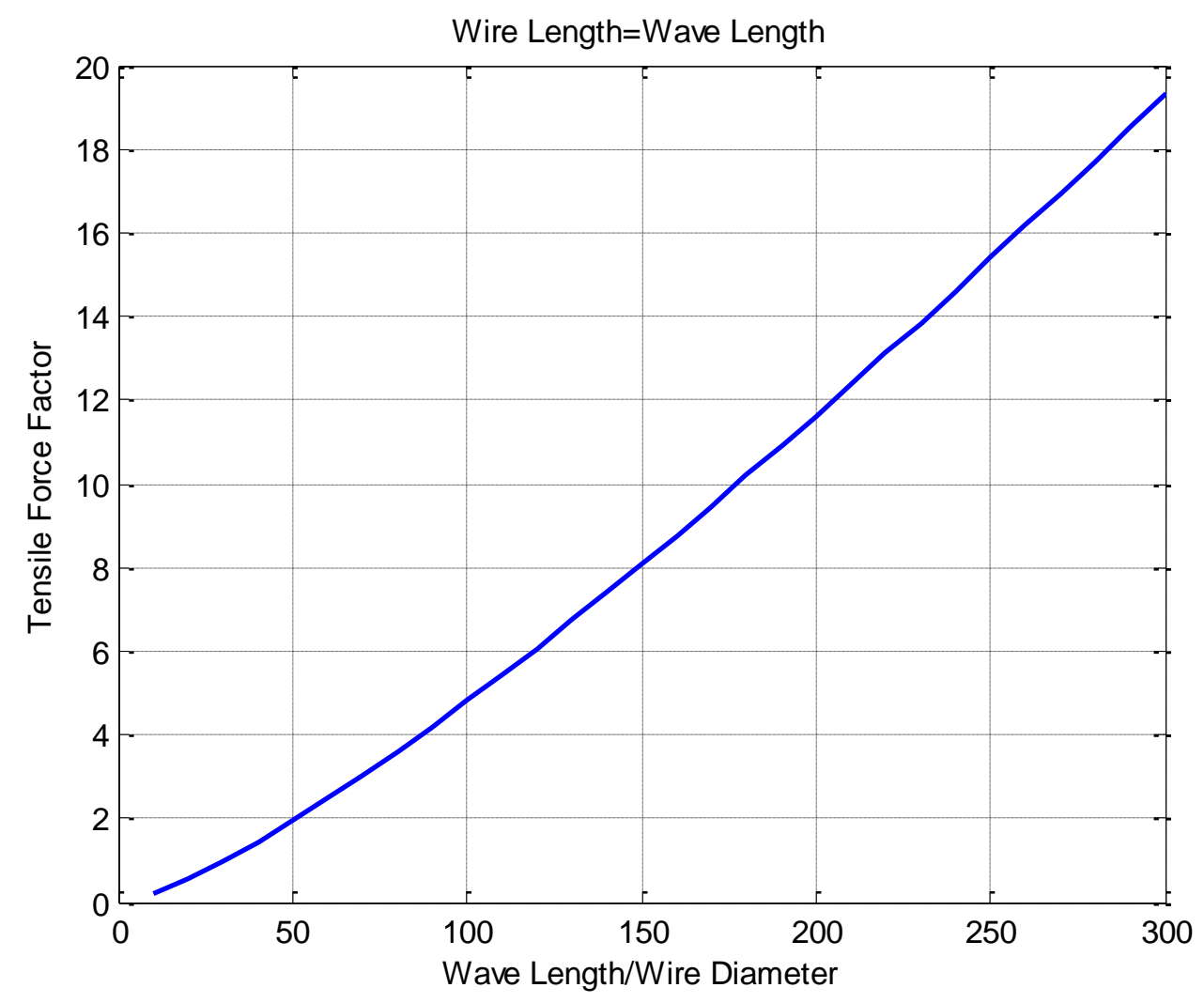

FIGURE 2. Tensile force factor as function of the ratio of the wire length (equal to transient wave length of first harmonic) divided by the wire diameter

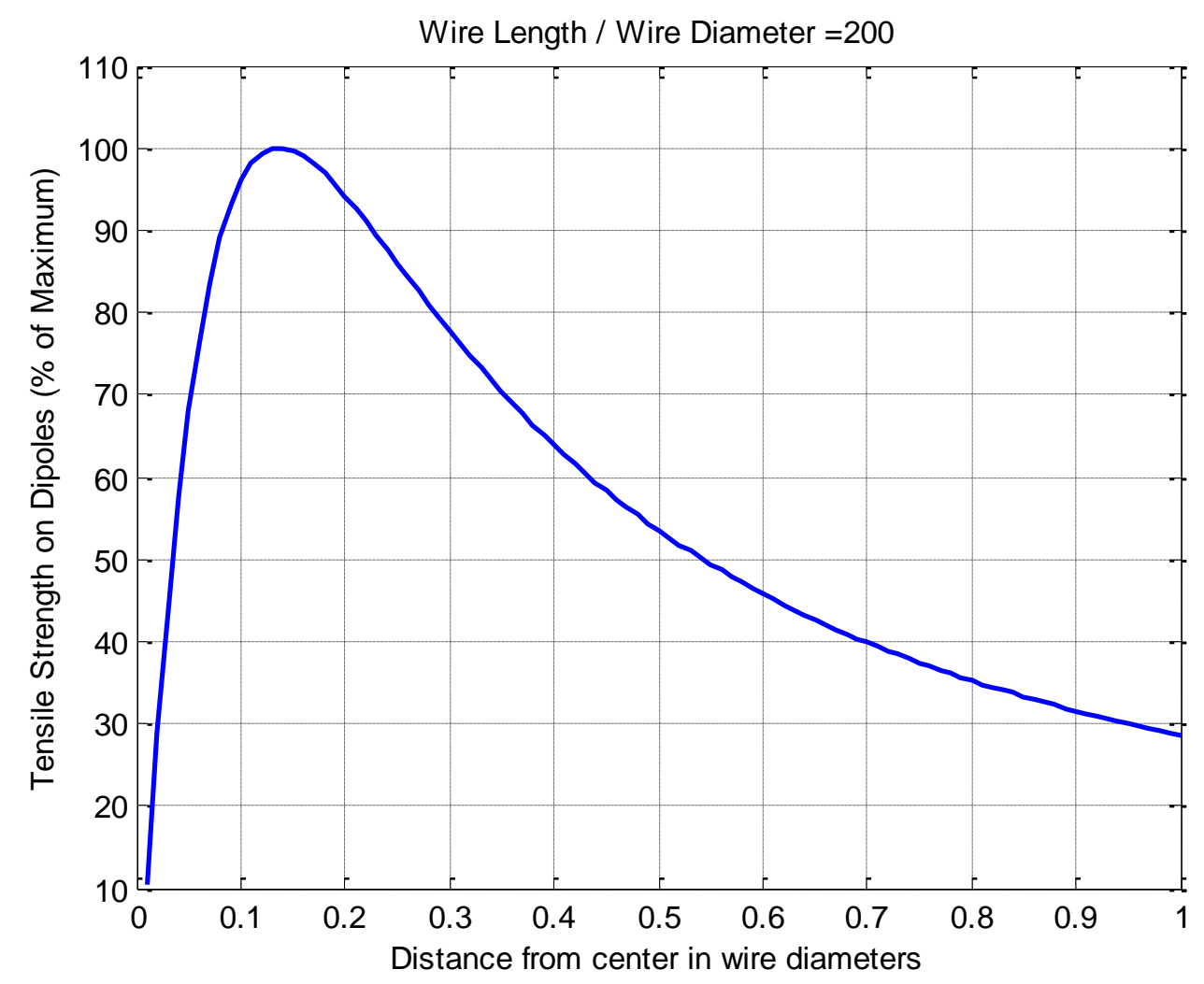


FIGURE 3.Tensile strength on the lower dipoles (arising by the action of upper dipoles) as function of their distance by the centre of the wire. Wire length 200 times the wire diameter.

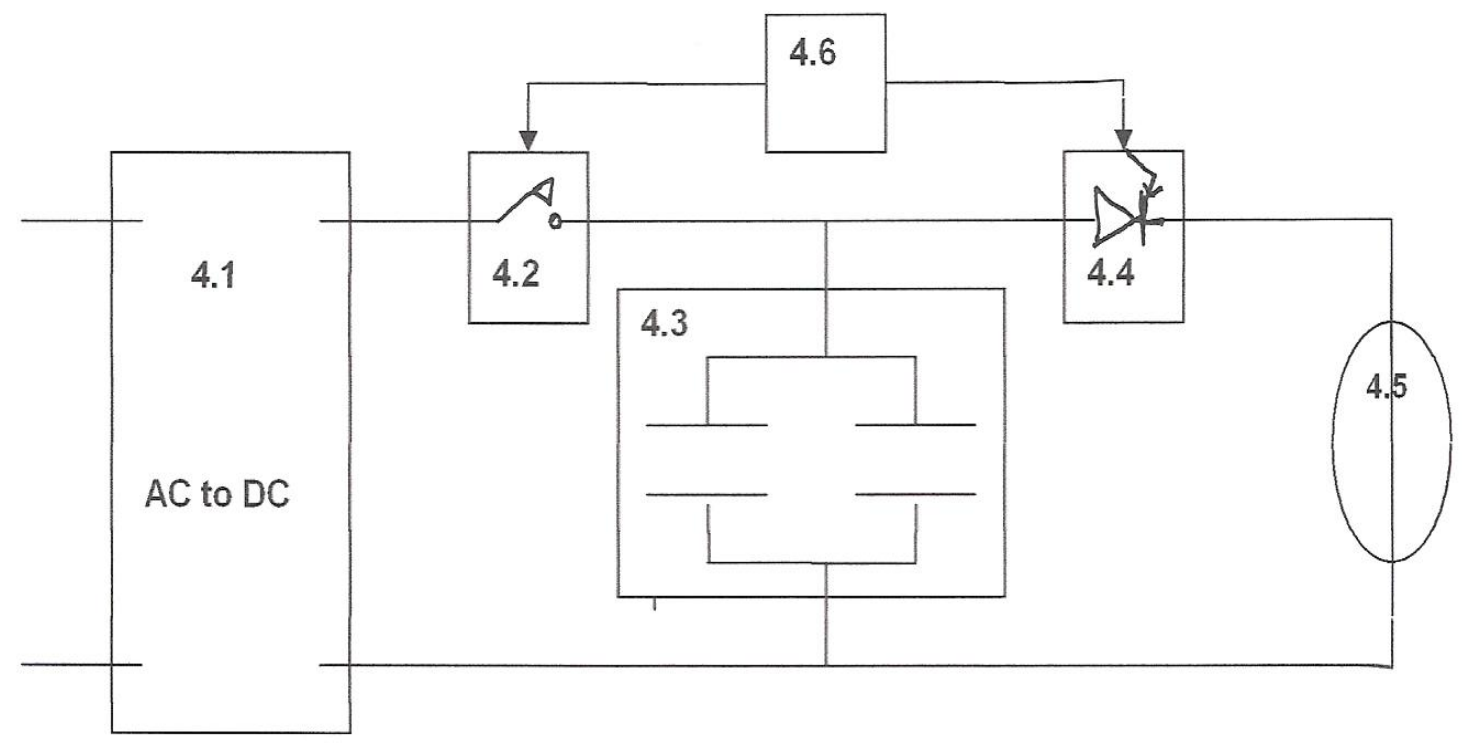

FIGURE 4. Schematic diagram of the circuit used in experiments : (4.1) AC/DC Inverter (4.2) Charging switch (4.3) Capacitor bank (4.4) Discharge thyristor (4.5) Testing wire (4.6) Electronic control unit.

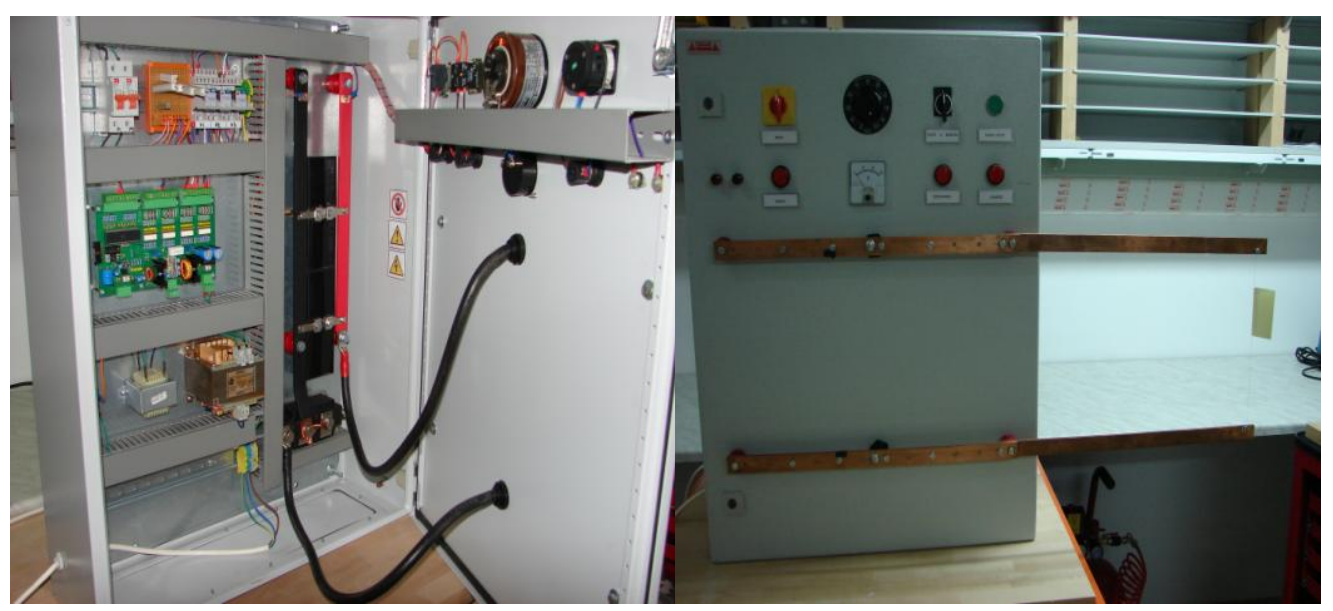


FIGURE 5. Exterior view of the whole device with the test wire attached (see white paper sign on the right)

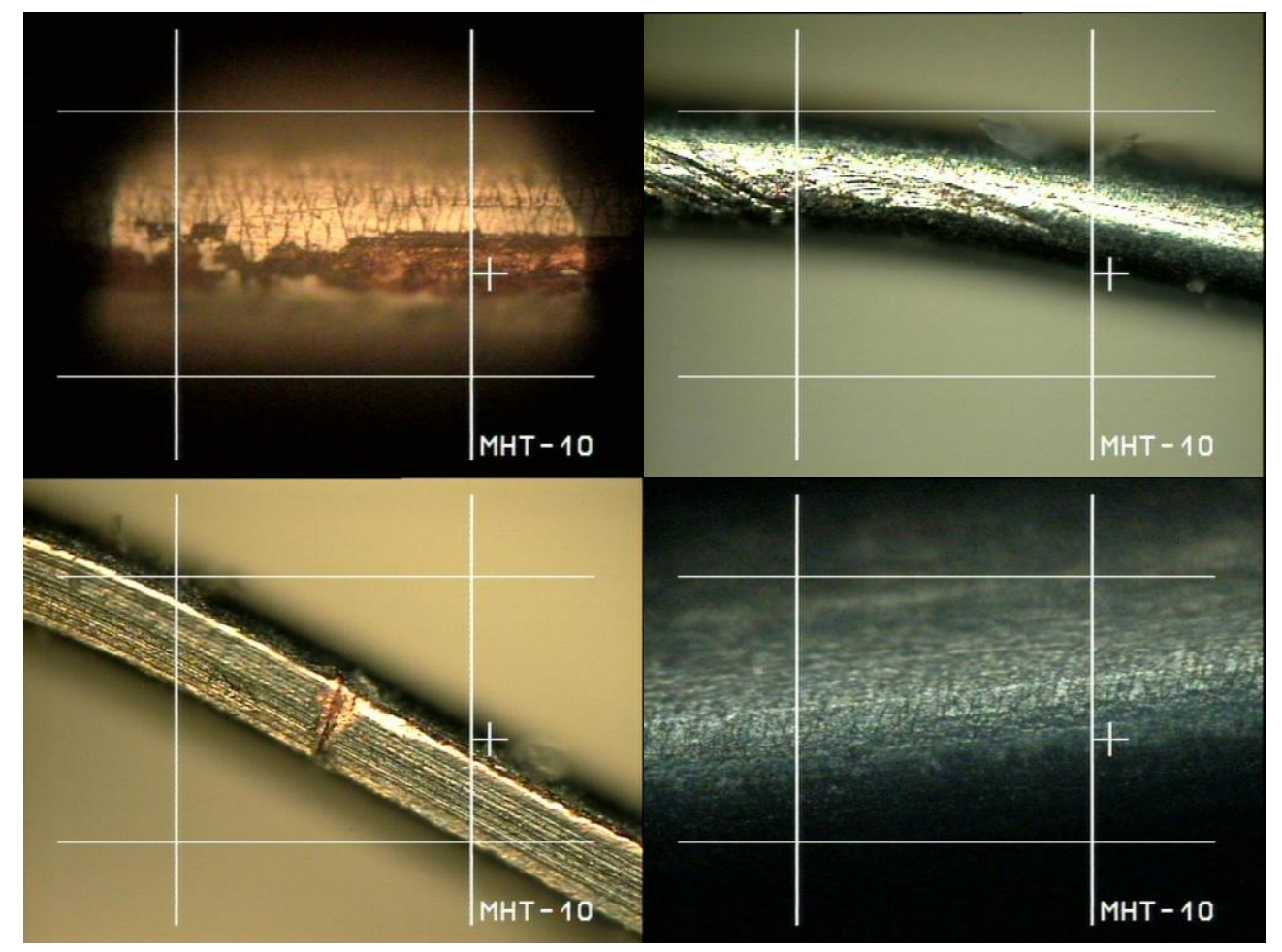

FIGURE 6. Condition of a wire surface after a succession of high power current pulses. 